\title{
MedienPädagogik
}

Zeitschrift für Theorie und Praxis der Medienbildung

Jahrbuch Medienpädagogik 17:

Lernen mit und über Medien in einer digitalen Welt

Herausgegeben von Klaus Rummler, Ilka Koppel, Sandra Aßmann,

Patrick Bettinger und Karsten D. Wolf

\section{Lernen mit und über Wikibooks}

\section{Erkenntnisse entwicklungsorientierter Fallstudien zur integrativen Medienbildung im Lehramtsstudium}

\author{
Franco Rau
}

\section{Zusammenfassung}

Die durch die Digitalisierung und Mediatisierung induzierten Transformationsprozesse stellen die Lehrerinnen- und Lehrerbildung in Deutschland vor eine Vielzahl von Herausforderungen (van Ackeren u.a. 2019). Wenngleich die Bedeutung exemplarischer Entwicklungsfelder zur hochschuldidaktischen Praxis sowie integrativen Medienbildung in der Schule bereits seit über 20 Jahren diskutiert werden (AG Erziehungswissenschaft 1997), besteht weiterhin ein Desiderat hinsichtlich der Gestaltung und wissenschaftlichen Begleitung verpflichtender Angebote zum Lernen mit und über Medien im Lehramtsstudium (Kammerl 2015, van Ackeren u.a. 2019). In einem entwicklungsorientierten Bildungsforschungsprojekt (Rau 2020) wurde daher gefragt, wie das Lernen mit Wikibooks im Rahmen bildungswissenschaftlicher Seminare Anlässe zum Lernen über öffentliche Wiki-Gemeinschaften eröffnen kann. Die Ergebnisse des Projektes zeigen, dass und wie die Realisierung einer integrativen $\mathrm{Me-}$ dienbildung im Lehramtsstudium gelingen kann. Die entwickelten Lehr- und Lernsituationen mit Wikibooks eröffneten vielfältige Möglichkeiten, um das Lernen mit und über soziale Medien zur medienpädagogischen Professionalisierung erfahrungs- und reflexionsbasiert zu fördern und zu unterstützen. Zudem können verschiedene Spannungsverhältnisse beschrieben werden, die sich bei der Realisierung eines Projektes zur integrativen Medienbildung ergeben. Der Beitrag gibt einen Einblick in die Konzeption des entwicklungsorientierten Bildungsforschungsprojektes und akzentuiert zentrale Erkenntnisse für die Forschung und Praxis. 


\title{
Media Education with Wikibooks. Insights of a Design Based Research Project
}

\begin{abstract}
The process of mediatization poses several challenges for teacher education in Germany (van Ackeren et al. 2019). For more than 20 years the importance of media education for schools and teacher education has been widely discussed (AG Erziehungswissenschaft 1997). So far comparatively few attempts have been made to establish media education as a compulsory part of teacher education and provide clear insights about new possibilities and limitations (e.g. Kammerl 2015, van Ackeren et al. 2019). A design-based research project (Rau 2020) therefore asked how learning with Wikibooks as a tool can open up opportunities for learning about wiki principles and the wiki community. The results of the project show that and how the implementation of integrative media education can succeed in teacher training. The developed learning situations with Wikibooks opened up a wide range of opportunities to promote and support learning with social media as a tool as well as social media as a topic. Besides, various tensions arise when implementing a project for integrative media education. The article gives an insight into the conception of the design-based research project and highlights key findings for research and practice.
\end{abstract}

\section{Lernen mit und über Medien als Entwicklungsperspektive}

Das Lernen mit und über digitale Medien erscheint in aktuellen bildungspolitischen Dokumenten als zentrale Leitformel für eine zeitgemässe «Bildung in der digitalen Welt» (KMK 2017). Die mit der Formulierung eines Lernens mit und über digitale Medien einhergehende Verknüpfung mediendidaktischer und medienerzieherischer Perspektiven wird auch unter den Begriffen einer «integrativen Medienbildung» (z.B. Kammerl und Atzeroth 2013, Aßmann und Herzig 2015), einer «integrativen Aufgabe» (z.B. BMBF 2010) sowie als integrativer Ansatz in den Unterrichtsfächern (z.B. AG Erziehungswissenschaft 1997, KMK 2017) diskutiert. Der Begriff der Integration soll in diesem Zusammenhang zum Ausdruck bringen, 
dass «neue Medien für die einzelnen Unterrichtsfächer eine grosse Bedeutsamkeit haben und zur Veränderung von fachbezogenen Lehr- und Lernprozessen beitragen» (Arbeitsgruppe Erziehungswissenschaft 1997, 45). In einem engen Verständnis von Integration stellt sich für die Unterrichtsfächer jeweils die Frage, inwiefern im Rahmen des fachlichen Lernens auch ein Lernen mit und ein Lernen über Medien stattfinden kann und soll. In einem weiten Verständnis von «Integration» wird davon ausgegangen, dass alle bildungsrelevanten Prozesse vor dem Hintergrund einer zunehmend digital geprägten Welt kritisch betrachtet und reflektiert werden müssen (AG Erziehungswissenschaft 1997, 45). In diesem Verständnis stellt sich auch für die universitäre Lehrerinnen- und Lehrerbildung die Frage, inwiefern im Rahmen der verschiedenen Studienbereiche ein Lernen mit und ein Lernen über digitale Medien stattfinden kann und soll.

Die universitäre Lehrerinnen- und Lehrerbildung in Deutschland bietet hinsichtlich der bereits vor über 20 Jahren aufgeworfenen Fragestellung noch heute viele Entwicklungsfelder (van Ackeren u.a. 2019). Dies zeigt sich auf curricularer Ebene u.a. daran, dass die Kritik verschiedener Bestandsaufnahmen (Kammerl und Mayrberger 2011, Kammerl und Ostermann 2010) hinsichtlich fehlender medienpädagogischer Elemente von der Kultusministerkonferenz in den Standards für Bildungswissenschaften erst in der im Jahr 2019 veröffentlichten Fassung (KMK 2019b) Berücksichtigung fand. Zugleich zeigt sich in Betrachtung der ländergemeinsamen inhaltlichen Anforderungen für die Fachwissenschaften und Fachdidaktiken (KMK 2019a) weiterhin, dass das Lernen über Medien nur in wenigen Fächern thematisiert wird. Ein weiteres Entwicklungsfeld stellt die hochschuldidaktische Praxis der Lehrerinnen- und Lehrerbildung dar. Seit Jahren wird regelmässig darauf hingewiesen, dass verpflichtende Angebote zu medienpädagogischen Professionalisierung für Lehramtsstudierende fehlen (Kammerl 2015, Kammerl und Ostermann 2010). Auch van Ackeren u.a. (2019, 111) betonen, dass «praxisorientierte Lerngelegenheiten und entsprechende (positive) Erfahrungen» für angehende Lehrkräfte «schon ab dem ersten Semester wichtig sind».

Zum Umgang mit dem skizzierten Praxisdefizit wurde an der TU Darmstadt im Jahr 2014 im Rahmen eines Dissertationsprojektes (Rau 2020) mit der Planung und Durchführung eines entwicklungsorientierten 
Forschungs- und Praxisprojektes zur integrativen Medienbildung für Lehramtsstudierende begonnen. Dabei zeigten sich zum Projektbeginn ähnliche Problembereiche: Zum einen standen praxisorientierte Gelegenheiten zum Lernen mit digitalen Medien nur Studierenden einzelner Studienfächer zur Verfügung. Zum anderen waren die Gelegenheiten zum Lernen über Medien bis zum Jahr 2017 nur als Wahloption im Lehramtsstudium der TU Darmstadt verankert. Die vorgefundene Praxis erschien daher als geeignetes Handlungs- und Forschungsfeld zur Durchführung eines entwicklungsorientierten Forschungsprojektes. Es wurde das Ziel verfolgt, im Rahmen bildungswissenschaftlicher Studienanteile Erfahrungsräume zu schaffen, in denen praxisorientierte Lerngelegenheiten mit und über soziale Medien einen integrativen Bestandteil darstellen.

Der vorliegende Beitrag gibt einen ersten Einblick in die konzeptionellen Überlegungen und präsentiert ausgewählte Erkenntnisse des Dissertationsprojektes (Rau 2020). Zu Beginn werden die Annahmen und Überlegungen zum Entwurf eines Seminarkonzeptes skizziert (Kap 2). Im Anschluss wird die Forschungsperspektive hinsichtlich der Fragestellung und dem methodischen Vorgehen konkretisiert (Kap. 3). In den folgenden Ergebnissen wird skizziert, inwiefern die entwickelten Lehr- und Lernsituationen mit Wikibooks Möglichkeiten eröffneten, um das Lernen mit und über soziale Medien zu fördern und zu unterstützen (Kap. 4). Abschliessend erfolgt eine Diskussion des Projektes (Kap. 5).

\section{Entwicklung eines Seminarkonzeptes zum Lernen mit und über Wikibooks}

Im Fokus des Konzeptes stehen die hochschuldidaktischen Handlungsebenen der «(Lern)Situationen» sowie der «(Lehr)Veranstaltungen» (Wildt 2002, 7). Zentrale Überlegungen zur Begründung der gewählten Zielperspektive sowie zum entwickelten Seminarkonzept werden im Folgenden skizziert. 


\subsection{Zielperspektiven}

Bei der Entwicklung eines Konzeptes wurden im Sinne einer integrativen Medienbildung drei Zielstellungen verfolgt: Das erste Ziel war die Förderung einer pädagogischen Artikulations- und Reflexionsfähigkeit im Rahmen der bildungswissenschaftlichen Studienanteile. Die zwei weiteren Ziele waren es, ein Lernen mit sowie ein Lernen über digitale soziale Medien zu ermöglichen. Im Fokus des vorliegenden Beitrages steht die zweite und dritte Zielperspektive.

Die Zielformulierung zur Ermöglichung eines Lernens mit digitalen sozialen Medien umfasst zwei Schwerpunkte. Die Gestaltung von Lehr- bzw. Lernsituationen mit digitalen sozialen Medien zur Unterstützung des fachlichen Lernens fokussiert die Entwicklung einer pädagogischen bzw. didaktischen Artikulations- und Reflexionsfähigkeit und markiert einen hochschuldidaktischen Schwerpunkt. In diesem Verständnis werden Medien als Mittel zur Unterstützung fachlicher Lernprozesse - in diesem Fall zur Anregung von Reflexionsprozessen sowie zur Auseinandersetzung mit erziehungswissenschaftlichen Modellen - verstanden. Diese Fokussierung erfolgt im Anschluss an das hochschuldidaktische Leitbild des «Wandels vom Lehren zum Lernen» (Wildt 2013, 40). Dem Lernen mit sozialen Medien wird in diesem Zusammenhang Potenzial zugesprochen, reflexive und partizipative Lernformate und -formen unterstützen zu können (Rau 2017b). Auf Basis empirischer Forschungsarbeiten ist zugleich bekannt, dass die Verwendung von digitalen sozialen Medien nicht automatisch zu entsprechenden Lernformen führt und insbesondere für Studierende eine Herausforderung darstellt (Grell und Rau 2011, Sim und Hew 2010). Zudem zeigt sich, dass die Verwendung sozialer Medien in institutionellen Lehrund Lernkontexten häufig im Kontext geschlossener Learning-Management-Systeme erfolgt. Möglichkeiten zur Öffnung klassischer Veranstaltungsformate durch die produktive Mitgestaltung öffentlicher Communities werden in der deutschsprachigen Diskussion überwiegend theoretisch diskutiert aber kaum empirisch erprobt und untersucht (Spannagel und Schimpf 2009). Als Orientierung dienen daher die englischsprachigen Fallstudien von Bonk u.a. (2009) und Xiao und Lucking (2008) zur Realisierung von studentischen Schreib- und Buchprojekten im Rahmen der WikibooksCommunity. 
Die Ermöglichung eines Lernens mit Medien dient zudem der medienpädagogischen Professionalisierung und zielt darauf ab, Lehramtsstudierenden Erfahrungen mit sozialen Medien zu ermöglichen und Anlässe zur Reflexion des eigenen Medienhandelns in Lehr- und Lernkontexten zu schaffen. Während von zukünftigen Lehrerinnen und Lehrern einerseits vielfältige medienpädagogische Kompetenzen und eine offene Haltung erwartet werden (z.B. Moser 2010, KMK 2017, 2019b), ist auf Basis empirischer Erhebungen anderseits bekannt, dass Lehramtsstudierende häufig über wenig Erfahrung verfügen und eine eher skeptische Haltung gegenüber digitalen Medien haben (Herzig und Grafe 2007). In Anlehnung an Schiefner-Rohs $(2012,44)$ wird davon ausgegangen, dass Hochschullehrende als Vorbilder zur Verwendung von Medien in Lehr- und Lernkontexten dienen können und «sich positive Erfahrungen mit Medien positiv auf die Einstellung zu Medien aus[wirken]». So eröffnet die erste Phase der Lehrerinnen- und Lehrerbildung die Chance, «dass an den bisherigen Einstellungen und Erfahrungen von Lehramtsstudierenden hinsichtlich digitaler Medien gearbeitet werden [kann]» (Schiefner-Rohs 2012, 44). Es wird davon ausgegangen, dass die Teilhabe an öffentlichen Wikigemeinschaften im Rahmen institutioneller Lehrveranstaltungen Studierenden potenziell neue Erfahrungen ermöglichen und so zur medienpädagogischen Professionalisierung beitragen kann.

Mit der Ermöglichung eines Lernens über digitale soziale Medien wird das grundlegende Ziel verfolgt, die Weiterentwicklung der eigenen $\mathrm{Me}$ dienkompetenz der Studierenden zu unterstützen und den Erwerb medienpädagogischer Kompetenzen zu ermöglichen. Diese Zielstellung orientiert sich an medienpädagogischen und bildungspolitischen Leitbildern (z.B. AG Erziehungswissenschaft 1997, KMK 2017). Im Fokus stehen dafür handlungsorientierte Angebote zur Teilhabe an einer partizipativen Medienkultur im Kontext öffentlicher Wikigemeinschaften. Mit dieser Schwerpunktsetzung wurde der Versuch unternommen, Diskussionen über Potenziale und gesellschaftliche Transformationsprozesse zur Teilhabe und Partizipation aufzugreifen (z.B. Biermann u.a. 2014, Mayrberger und Moser 2011). In Anlehnung an Mayrberger (2012), Mayrberger u.a. (2013) und Moser (2010) wurde davon ausgegangen, dass der professionelle Umgang mit sozialen Medien auch neue Fähigkeiten und Wissensbestände 
von Lehrkräften erfordert. In Adaption von Tulodziecki u.a. (2013) wurden dafür die folgenden Aspekte als relevant betrachtet: das Gestalten und Verbreiten eigener Wiki-Beiträge, das Durchschauen und Beurteilen von Bedingungen der Wissensproduktion in öffentlichen Wikigemeinschaften am Beispiel von Wikibooks sowie die Fähigkeit zur begründeten Auswahl und der sachgerechten Nutzung von sozialen Medien zum Lernen und Zusammenarbeiten.

\subsection{Skizze des Blended Learning Konzeptes}

Der Entwurf des Seminarkonzeptes orientierte sich u.a. an den medienpädagogischen und mediendidaktischen Prinzipien der «Produkt- und Projektorientierung» (Rau 2020), der «Kooperation, Kollaboration und Kommunikationsorientierung» (ebd.) und zielt auf die «Eröffnung von Partizipationsmöglichkeiten und Erfahrungsorientierung» (ebd.) ab. Es wurde ein dreiphasiges Blended-Learning-Konzept zur kollaborativen Erstellung eines Wikibooks mit Studierenden entworfen. Die drei Phasen umfassten die folgenden Elemente:

- Bewusstwerdung eigener Vorstellungen: Entwicklung von Metaphern zur Artikulation von (alltagsbezogenen) Vorstellungen der Studierenden zu pädagogischen Grundbegriffen und Handlungen. Verschriftlichung der in Gruppenarbeiten verdichteten Metaphern zu Wikiseiten.

- Erarbeitung pädagogischer Perspektiven: Erarbeitung bildungswissenschaftlicher Modelle und Perspektiven durch die Auseinandersetzung mit Literatur als spätere Analysefolie für die selbst entwickelten Metaphern (aus Phase I). Erstellung von Wikiseiten und Diskussion im Seminar.

- Kriteriengeleitete Reflexion eigener Vorstellungen: Erstellung von (individuellen) Analysen der eigenen Metaphern (aus Phase I) mithilfe ausgewählter Kriterien der erarbeiteten bildungswissenschaftlichen Modelle und Perspektiven (aus Phase II) in Form von eigenen Texten. 


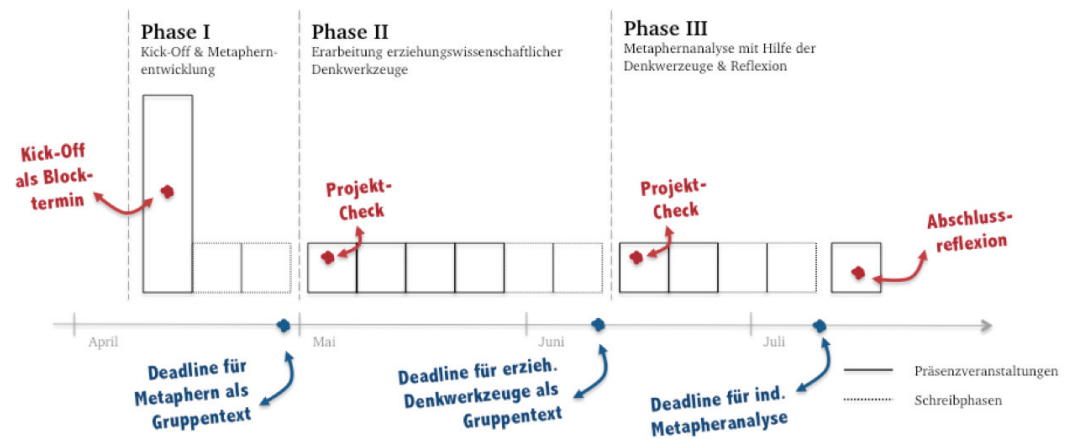

Abb. 1.: Visualisierung des Veranstaltungsverlaufs in drei Phasen.

Zur Verfolgung der skizzierten Zielperspektiven wurde eine produktive Auseinandersetzung mit Wikibooks als exemplarische, wikibasierte Sharing-Community angestrebt. Um den Studierenden vielfältige Erfahrungen zur Gestaltung eigener Wikibeiträge zu ermöglichen, wurden sie mit gruppenbezogenen und individuellen Gestaltungsaufgaben in jeder Phase konfrontiert. Zudem wurde davon ausgegangen, dass die Mitarbeit an einer öffentlichen Sharing-Community die Möglichkeit bietet, die Voraussetzungen und Bedingungen öffentlicher Wissensproduktion in Wikis erfahren zu können. Im Projekt wurde zudem der Versuch unternommen, den Studierenden kollaboratives Lernen durch die Nutzung sozialer Medien zu ermöglichen und zugleich ihre Fähigkeit zu fördern, entsprechende mediale Angebote zu nutzen. Um die Zusammenarbeit der Studierenden zu unterstützen, wurde Ihnen neben der Plattform Wikibooks auch ein geschlossener Moodle-Kurs mit verschiedenen Funktionen zum Austausch zur Verfügung gestellt.

\section{Lernen mit und über Medien als Forschungsperspektive}

Das Lernen mit und über digitale Medien eröffnet neben der skizzierten Entwicklungsperspektive für die Praxis nach Petko (2011) auch eine interessante Forschungsperspektive. In Form von praxisorientierter Forschung bietet sich die Möglichkeit, medienpädagogische und mediendidaktische Perspektiven stärker aufeinander zu beziehen, um Erkenntnisse über «den Anregungsgehalt medienpädagogischen Handelns» (Petko 2011, 248) zu 
gewinnen. An dieser Perspektive anknüpfend, werden im Folgenden die Fragestellungen des Projektes vorgestellt und das forschungsmethodische Vorgehen skizziert.

\subsection{Forschungsperspektive und Fragestellung}

In den vergangenen Jahren wurde von verschiedenen Positionen ein Plädoyer für mehr praxisorientierte Forschung in der Medienpädagogik und Mediendidaktik formuliert (Spanhel 2007, Petko 2011, Niesyto 2014). Petko (2011) betont in diesem Zusammenhang die Möglichkeit, die Perspektiven der Medienpädagogik und Mediendidaktik aufeinander beziehen zu können. Der gemeinsame Fokus ist die Frage, «wie Menschen mit Medien für Medien sensibilisiert werden können» (ebd., 248). Dieser Argumentation folgend, bedeutet medienpädagogisch zu forschen insbesondere, «innovative medienpädagogische Praxisprojekte zu entwickeln, sie auszuprobieren, zu überprüfen und zu revidieren» (ebd.).

An dieser Überlegung anknüpfend, wurde mit der Durchführung eines entwicklungsorientierten Forschungsprojektes nach Reinmann und Sesink (2014) das Ziel verfolgt, einen praxis- und forschungsbezogenen Beitrag zur integrativen Medienbildung in der Lehrerinnen- und Lehrerbildung für die bildungswissenschaftlichen Studienanteile vorzulegen. In Anlehnung an Petko (2011) erfolgte die Verknüpfung einer medienpädagogischen und einer mediendidaktischen Perspektive, indem über die innovative Gestaltung von Lehr- und Lernsituationen mit sozialen Medien untersucht wurde, inwiefern Studierende für soziale Medien sensibilisiert werden können. Im Sinne einer integrativen Medienbildung wurde darüber hinaus das fachliche Lernen in den bildungswissenschaftlichen Studienanteilen als Entwicklung einer pädagogischen Artikulations- und Reflexionsfähigkeit berücksichtigt. Die leitende Fragestellung des Projektes lautete: Inwiefern kann die Mitgestaltung eines öffentlichen Wikibooks in erziehungswissenschaftlichen Seminaren in der Lehrerinnen- und Lehrerbildung Situationen schaffen, um eine pädagogische Artikulations- und Reflexionsfähigkeit zu entwickeln und ein Lernen mit und über soziale Medien anzuregen? Zur empirischen Bearbeitung wurde die Fragestellung weiter differenziert. Im vorliegenden Beitrag werden die zwei folgenden Teilfragen diskutiert: 
- Inwiefern ist es mit den entworfenen Lernsituationen im Rahmen der Seminare gelungen, Studierenden neue Erfahrungen zur Verwendung sozialer Medien in der Lehre zu ermöglichen?

- Inwiefern eröffnet die Mitgestaltung eines öffentlichen Wikibooks im Rahmen bildungswissenschaftlicher Seminare Lern- und Reflexionsanlässe zum Lernen über soziale Medien?

\subsection{Methodisches Vorgehen}

In einem zirkulären Vorgehen erfolgte die Untersuchung von Veränderungs- und Entwicklungspotenzialen auf einer mikrodidaktischen Ebene. Das Vorgehen umfasst nach Sesink und Reinman (2015) die wechselseitig aufeinander bezogenen Elemente zur Problematisierung der aktuellen Praxis, zur Formulierung eines theoretisch begründeten Entwurfs, empirische Erprobungen, wissenschaftliche Analysen der konkreten Handlungspraxis sowie eine Interpretation und Diskussion der erarbeiteten Ergebnisse (Abb. 1). Das theoretisch begründete Seminarkonzept wurde als universitäre Lehrveranstaltung in den Modulen «Didaktik, Methodik und Medien» und «Grundlagen pädagogischen Denkens und Handelns» über zwei Semester an der TU Darmstadt mit 111 Studierenden erprobt, analysiert, modifiziert und hinsichtlich der formulierten Fragestellung ausgewertet.
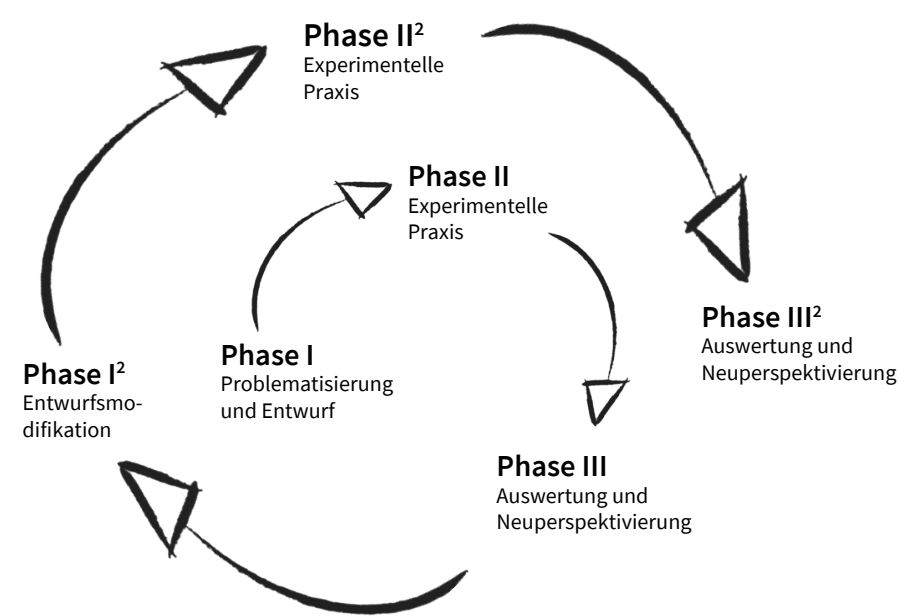

Phase III

Auswertung und

Neuperspektivierung

Abb. 2.: Realisierte Forschungsphasen nach Sesink und Reinmann (2015). 
Die formulierten Teilfragen markieren zentrale Orientierungen für die empirische Analyse. Zur Beantwortung der Fragestellungen erfolgte eine Triangulation unterschiedlicher Perspektiven (Flick 2011, 13 ff.). Drei Blickwinkel wurden fokussiert: die Perspektive der Studierenden, die Perspektive der Lehrenden sowie eine objektorientierte Perspektive. Ausgewertet wurden quantitative Fragebögen $(n=32)$ sowie Gruppengespräche $(n=7) \mathrm{im}$ Rahmen der durchgeführten Veranstaltungsevaluationen, Interviews mit Studierenden ( $n=18)$, Sprachmemos in der Rolle als Lehrender $(n=56)$ sowie Bearbeitungsstatistiken des Wikibooks und des Moodlekurses (ausführlich in Rau 2020). Während die Auswertung der qualitativen Daten in Form von zusammenfassenden und strukturierenden Inhaltsanalysen (Mayring 2002, Kuckartz u.a. 2008) erfolgte, wurden Verfahren der deskriptiven Statistik zur Auswertung der quantitativen verwendet (Kühnel und Krebs 2006). Das Vorgehen orientiert sich an Qualitäts- und Prozessstandards einer entwicklungs- und gestaltungsorientierten Bildungsforschung (Tulodziecki u.a. 2013, Sesink und Reinmann 2015).

\section{Zentrale Ergebnisse}

Die Ergebnisse des entwicklungsorientierten Bildungsforschungsprojektes sind vielschichtig und werden im Folgenden akzentuierend zusammengefasst. Auf Basis unterschiedlicher empirischer Indikatoren kann gezeigt werden, dass die entwickelten Lehr- und Lernsituationen mit Wikibooks vielfältige Chancen eröffnen, das Lernen mit sozialen Medien erfahrungsbasiert zu unterstützen. Es werden wahrgenommene Lerngelegenheiten zum kooperativen, zum partizipativen sowie reflexiven Lernen skizziert. Zudem wird gezeigt, inwiefern es mit dem entwickelten Seminarkonzept gelungen ist, das Lernen über soziale Medien zur medienpädagogischen Professionalisierung erfahrungs- und reflexionsbasiert zu fördern und zu unterstützen. Abschliessend werden neue Handlungsspielräume und Spannungsverhältnisse in der Hochschullehre skizziert, die sich bei der Realisierung eines Projektes zur integrativen Medienbildung mit Wikibooks ergeben. 


\subsection{Potenziale zum Lernen mit digitalen sozialen Medien}

Für mehrere Studierende wurden die entwickelten Lernsituationen zum Ausgangspunkt kooperativer und kollaborativer Zusammenarbeit und ermöglichten neue Erfahrungen zur Verwendung sozialer Medien in einem institutionellen Kontext. Die Sprachmemos aus der Perspektive des Lehrenden sowie die Nutzungsstatistiken der Moodle-Plattform zeigen, dass studentische Arbeitsgruppen im Rahmen der ersten Erprobung interne Moodle-Foren zum Austausch sowie zum gemeinsamen Schreiben nutzten. In den Interviews wurden von Studierenden in diesem Kontext verschiedene Strategien der Zusammenarbeit beschrieben, die über die zur Verfügung gestellten Medien hinausgehen. In der ersten Iteration erfolgte das kollaborative Schreiben beispielsweise von mehreren Arbeitsgruppen in Form lokal gespeicherter Textverarbeitungsdokumente, welche per EMail versendet wurden. Unabhängig von der verwendeten Strategie und den verwendeten Medien waren die entwickelten Aufgabenstellungen jeweils der Ausgangspunkt der Zusammenarbeit. Über das gemeinsame Bearbeiten des Wikibooks sowie Schreibzeiten im Rahmen der Präsenzveranstaltungen gelang es in der zweiten Iteration, dass die Studierenden das Wiki als Werkzeug zum kollaborativen Schreiben nutzten. Die Analyse der Bearbeitungsstatistik zeigt beispielsweise, dass in der zweiten Iteration die Mehrheit der Studierenden als Autorinnen und Autoren der Gruppentexte aktiv wurden und im Vergleich zur ersten Iteration überwiegend mehr Bearbeitungen durchgeführt haben.

Möglichkeiten zur Partizipation konnten im Rahmen der zwei Iterationen in unterschiedlicher Weise realisiert werden. In der ersten Iteration wurden Partizipationsmöglichkeiten und -verpflichtungen hinsichtlich der Mitgestaltung des Wikibooks eröffnet. Neben der Verpflichtung zum Schreiben von Texten für das Wikibook hatten die studentischen Arbeitsgruppen im Rahmen der ersten Erprobung Freiheiten zur Organisation der eigenen Zusammenarbeit, zur Wahl thematischer Schwerpunkte sowie zur Gestaltung der eigenen Wikiseiten. Im Verlauf des Seminars wurden mit den Studierenden und unter Berücksichtigung der Wikibook-Community formal einheitliche Gestaltungsleitlinien entwickelt. Die Entscheidung erfolgte durch gemeinsame Diskussionen sowie mithilfe von OnlineAbstimmungen. Die Formen der Beteiligungen lassen sich in Anlehnung 
an Mayrberger $(2013,169)$ auf der Ebene der Mitbestimmung verorten. Auf Basis der Analyse der ersten Fallstudie sowie des modifizierten Entwurfs gelang es in der zweiten Iteration u.a., Studierenden weitere Mitbestimmungsmöglichkeiten zur methodischen Organisation der zweiten Seminarphase einzuräumen. Die benannten Partizipationschancen wurden von Studierenden in den Veranstaltungsevaluationen positiv hervorgehoben (Rau 2020).

Die entwickelten Lernsituationen eröffneten auch Anlässe zur Reflexion des Lernens mit digitalen Medien in institutionellen Vermittlungssituationen. Beispielsweise wurden von den Studierenden in den Interviews und in den Gruppengesprächen das eigene Medienhandeln im Umgang mit öffentlichen Wikis hinterfragt und reflektiert. Die gemeinsame Gestaltung eines Wikibookprojektes in der Hochschullehre ermöglichte für verschiedene Studierende auch neue Möglichkeiten zum Einsatz digitaler Medien in der Schule zu erkennen bzw. vertiefend kennenlernen zu wollen. So wünschten sich Studierende in der ersten Fallstudie jenseits der curricular verankerten Seminarthemen mehr über die Einsatzmöglichkeiten von digitalen Medien in der Schulpraxis zu erfahren. In der zweiten Fallstudie wurde die Mitgestaltung des Wikibooks als lohnenswerte Erfahrung für die spätere Schulpraxis markiert. Diese Ergebnisse lassen sich als empirische Indikatoren dafür deuten, dass das realisierte Veranstaltungskonzept für die Studierenden als «Vorbild» (Schiefner-Rohs 2012, 45) für den Einsatz digitaler Medien dient und positive Wirkungen auf ihre Einstellungen zu digitalen Medien entfalten kann.

\subsection{Potenziale zum Lernen über digitale soziale Medien}

Auf Basis der empirischen Erprobungen und wissenschaftlichen Analyse wird im Folgenden gezeigt, inwiefern die entwickelten Lernsituationen von Studierenden als Anlässe individueller Entwicklungsprozesse wahrgenommen wurden. Im Fokus der Diskussion stehen drei Dimensionen: Gestalten und Veröffentlichen eigener Wikibeiträge, Durchschauen und Beurteilen von Bedingungen der Wissensproduktion und Verbreitung in öffentlichen Wikis sowie Auswahl und Nutzen von sozialen Medien zur Kooperation und zum Lernen. 


\subsubsection{Gestalten und Veröffentlichen eigener Wikibeiträge}

Auf Basis der Interviewauswertung der ersten Iteration kann exemplarisch gezeigt werden, dass die Wahrnehmung von und Auseinandersetzung mit Aufgaben zur Gestaltung von Wikiseiten für Studierende zum Ausgangspunkt individueller Entwicklungsprozesse wurde. Verschiedene Studierende, welche jeweils ihre eigene Medienkompetenz skeptisch betrachteten, beschrieben die Gestaltung und Veröffentlichung eines Wikibeitrages als Herausforderung. Dabei ermöglichte die individuelle Auseinandersetzung mit dem Wiki-Editor und der Wikisyntax Studierenden Lernerfahrungen und nahm ihnen die Angst vor der Erstellung eigener Wikibeiträge. Diese Erfahrungen werden beispielsweise als «cool» und zufriedenstellend beschrieben: «Okay wir haben es jetzt hingekriegt, dass jetzt wirklich im Internet was von uns steht» (Bog, 18). Die gelungene Erstellung und Begleitung öffentlicher Wikiseiten wurden in beiden Iterationen von Studierenden als neue Erfahrung beschrieben, welche das Kennenlernen medialer Gestaltungsmöglichkeiten ermöglichte.

Interessant erscheint dabei, dass der herausfordernde Charakter der Aufgabenstellung in der zweiten Iteration nur von wenigen Studierenden markiert wurde. Stattdessen betonten Studierende, dass die bereits vorhandenen Beiträge als hilfreiche Orientierung bei der eigenen Gestaltung von Wikitexten dienten. Diese Ergebnisse spiegeln sich auch in der quantitativen Veranstaltungsevaluation. Insbesondere in der zweiten Iteration, in der sich mehr Studierende an der Gestaltung des Wikibooks beteiligten, zeigte sich, dass das Mitschreiben an einem Wikibook verschiedenen Studierenden mehr Sicherheit gab, sich aktiv an der Gestaltung von Wikis zu beteiligen. Die praktische Erstellung von Textprodukten, welche durch die Veröffentlichung auch von Menschen ausserhalb des Seminars wahrgenommen wurden, wurde in den Gruppengesprächen von verschiedenen Studierenden als positive Erfahrung hervorgehoben. 


\subsubsection{Durchschauen und Beurteilen von Bedingungen der Wissensproduktion und Verbreitung in öffentlichen Wikis}

Konkrete Anlässe zur Thematisierung und Auseinandersetzung mit den Bedingungen der Wissensproduktion und Verbreitung in öffentlichen Wikis zeigen sich u.a. in den Praxisreflexionen sowie der Analyse der Versionsgeschichte des Wikibooks. Die Beteiligung von Wikibookianerinnen und Wikibookianern wurde für Studierende u.a. in Form von Kommentaren auf Diskussionsseiten sowie durch Überarbeitungen von Wikitexten sichtbar. Aus der Perspektive des Lehrenden eröffneten sich so authentische Möglichkeiten, um Prinzipien der «Partizipation» und der «Prozessualität» von Wikis in den Präsenzveranstaltungen zum Gegenstand zu machen (Iske und Marotzki 2010). Die Verwendung von Visualisierungen und Schemata eröffnete zudem die Thematisierung rechtlicher Rahmenbedingungen öffentlicher Wikitexte und ermöglichte so potenzielle Lernanlässe für Studierende.

Die Ergebnisse der Veranstaltungsevaluation sowie der Interviews zeigen, dass die Interaktionen mit Wikibookianerinnen und Wikibookianern für verschiedene Studierende zu einem zentralen Thema der Veranstaltung und zum Ausgangspunkt individueller und kollektiver Reflexionen wurden. So war es für mehrere Studierende zunächst überraschend, dass die erstellten Wikitexte von Menschen ausserhalb des Seminars gelesen und kommentiert wurden. Zum Abschluss des Seminars hinterfragten verschiedene Studierende die fachliche Expertise der Wikibookianerinnen und Wikibookianer und problematisierten die Art und Weise der von ihnen formulierten Rückmeldungen. Die Bewertung der Veränderbarkeit fremder sowie eigener Beiträge wurde hinsichtlich verschiedener Gesichtspunkte diskutiert. Die Möglichkeit, die Veränderungen von Wikiseiten über die Versionsgeschichte nachvollziehen zu können, war der Mehrheit der Studierenden neu. 


\subsubsection{Auswahl und Nutzen von sozialen Medien zum Schreiben und Lernen}

Exemplarisch zeigen Erzählungen und Reflexionen der Interviews in beiden Iterationen, dass die bereitgestellten Bedingungen für verschiedene Studierende einen Anstoss für individuelle Entwicklungsprozesse darstellten. In einem Interview wurde beispielsweise die zu Beginn gewählte Schreibstrategie, einen Text in einem Textverarbeitungsprogramm zu formulieren und bei jeder Änderung an die Gruppenteilnehmerinnen und -teilnehmer per E-Mail zu versenden, rückblickend problematisiert und mit den Möglichkeiten zur kollaborativen Texterstellung mit Hilfe eines Wikis verglichen. In der Reflexion dieser Erfahrung geht die interviewte Person davon aus, dass die Nutzung des Wiki-Editors für die Zusammenarbeit die bessere Alternative gewesen wäre. In der zweiten Fallstudie thematisierten und reflektierten die Studierenden die Möglichkeiten unterschiedlicher Anwendungen zur Zusammenarbeit. In ihren Erzählungen differenzierten die Studierenden die unterschiedliche Eignung von Anwendungen für bestimmte Einsatzzwecke und diskutierten die Erprobung weiterer Angebote (z.B. Etherpad und Instant-Messenger).

Weitere Impulse zur Auswahl sowie zum Nutzen von sozialen Medien zum gemeinsamen Schreiben und Lernen eröffneten die Rückmeldungen zu den studentischen Arbeitsprodukten. Neben den inhaltlichen Anregungen konnten die Rückmeldungen der Lehrenden den Studierenden neue Möglichkeiten zur Nutzung digitaler Medien aufzeigen. Diese Einsicht wurde u.a. von Studierenden in Interviews und Gruppengesprächen der zweiten Fallstudie thematisiert. Darüber hinaus wurden die Rückmeldungen in den Veranstaltungsevaluationen jeweils positiv von den Studierenden bewertet.

\subsection{Neue Handlungsspielräume und -grenzen für die Hochschullehre}

Mit der gemeinsamen Gestaltung eines Wikibooks in Form eines Projektseminars, in dem digitale Medien in vielfältigen Situationen von Bedeutung sind, konnten die teilnehmenden Studierenden im Sinne von Spannagel (2013) eine Vielzahl neuer Lernerfahrungen im Lehramtsstudium 
machen. Insbesondere in den Gruppengesprächen der ersten und zweiten Iteration hoben die Studierenden hervor, dass die Veranstaltung für sie kein «08/15-Seminar» war und von ihnen als positive Abwechslung zu anderen Veranstaltungsformaten der bildungswissenschaftlichen Studienanteile erlebt worden ist. Die Einschätzung begründeten die Studierenden hinsichtlich spezifischer Lernsituationen, mit Bezug auf die Struktur des gesamten Seminarkonzeptes sowie des Medieneinsatzes zum öffentlichen Schreiben. Die gemeinsame Gestaltung eines Wikibooks in Form des vorgelegten Entwurfes kann für Hochschullehrende als Beispiel dienen, wie der Einsatz von sozialen Medien in der Hochschullehre zur integrativen Medienbildung erfolgen kann und zur Erweiterung hochschuldidaktischer Vielfalt in Veranstaltungen der Lehrerinnenbildung und Lehrerbildung beitragen kann.

Zugleich können auf Basis der Erkenntnisse der zwei Iterationen auch Gestaltungsgrenzen von Lerngelegenheiten mit Wikibooks für die Hochschullehre spezifiziert werden. Für die gemeinsame Erstellung eines Wikibooks im Rahmen eines Seminares erfolgt dies im Folgenden für das Spannungsverhältnis von «Offenheit oder didaktische Vorstrukturierung» (Rau 2020). Die Einbindung des Wikibooks erfolgte in den zwei entwickelten und erprobten Entwürfen auf unterschiedliche Weise. Mit der Modifikation des vorgestellten Entwurfs veränderte sich auch der Schwerpunkt studentischer Lern- und Reflexionsanlässe. Die offene Herangehensweise der ersten Fallstudie zur Erstellung von Wikiseiten wurde von verschiedenen Studierenden als Herausforderung beschrieben. Sofern die Studierenden diese Herausforderungen annahmen, wurde die Gestaltung eigener Wikibeiträge zum Ausgangspunkt individueller Entwicklungsprozesse. Zugleich entstanden im Prozess der Erstellung des Wikibooks Probleme hinsichtlich der Formatierung der Texte sowie der angemessenen Verlinkung im Wikibook. Diese Probleme eröffneten neue Lernanlässe zur Thematisierung der sachgerechten Nutzung eines Wiki-Editors. Zudem wurden diese Probleme von Wikibookianerinnen und Wikibookianern öffentlich kommentiert und überarbeitet. Diese Interaktionen konnten im Rahmen der Präsenztermine als Prinzipien und Bedingungen öffentlicher Wissensproduktion thematisiert und zu einem Ausgangspunkt weiterer Entwicklungsprozesse für Studierende werden. 
Mit der gemeinsamen Bearbeitung von Wikibeiträgen in den Präsenzveranstaltungen wurde in der zweiten Erprobung der Versuch unternommen, die entstandenen Probleme zu verringern und mehr Studierende dabei zu unterstützen, eigene Wikibeiträge zu gestalten und zu veröffentlichen. Dies erfolgte u.a. durch die Bereitstellung von weiteren Orientierungshilfen zur Gestaltung und Veröffentlichung eigener Wikitexte. Die Analyse der Versionsgeschichte sowie der Ergebnisse der Interviewauswertung zeigen, dass sich in der Folge mehr Studierende aktiv an der Mitgestaltung des Wikibooks beteiligten und die Erstellung von Beiträgen deutlich seltener als zuvor als Problem beschrieben wurde. Durch die sachgerechte Nutzung der Wikisyntax verringerte sich die Anzahl an Problemen bei der Erstellung und Bearbeitung von Wikiseiten. Gleichzeitig verringerte sich auch die Anzahl an Interaktionen mit Wikibookianerinnen und Wikibookianern, da deutlich weniger Korrekturen notwendig waren. So gab es in der zweiten Erprobung nur wenige Möglichkeiten, die Auseinandersetzung mit der Wikibookcommunity zum Thema in den Präsenzveranstaltungen zu machen. Dies scheint ein zentraler Grund dafür zu sein, dass die Interaktionen mit Wikibookianerinnen und Wikibookianern in der zweiten Iteration nur von wenigen Studierenden wahrgenommen wurden.

Im Zusammenhang mit den Veränderungen zeigte sich, dass die Studierenden unterschiedliche Erfahrungen machen konnten und die Auseinandersetzung mit dem Wikibook zum Ausgangspunkt unterschiedlicher Entwicklungsprozesse wurde. Interessant ist dabei das Ergebnis, dass in beiden Fallstudien ein anderer Schwerpunkt zu erkennen ist. Damit wird auch sichtbar, dass es nicht gelang Lernsituationen zu entwickeln, in denen die zwei Zieldimensionen «Gestalten und Veröffentlichen eigener Wikibeiträge» und «Durchschauen und Beurteilen von Bedingungen der Wissensproduktion und Verbreitung in öffentlichen Wikis» in gleicher Weise adressiert werden konnten. Auf Basis der vorgestellten Ergebnisse ist vielmehr die Annahme begründet, dass im Rahmen des Wikibookprojektes erst durch formale Probleme, die durch die nicht sachgerechte Gestaltung eigener Wikibeiträge entstanden, das Durchschauen und Beurteilen von Bedingungen der Wissensproduktion über Wikibooks möglich wurde. Mit der Formulierung «Offenheit oder didaktische Vorstrukturierung» wurde versucht, dieses Spannungsfeld zum Ausdruck zu bringen. Für die 
Entwicklung neuer Handlungsspielräume erscheint die Frage zentral, wie der Umgang mit Fehlern als produktive Lern- und Entwicklungsanlässe berücksichtigt werden kann.

\section{Perspektiven für Forschung und Praxis}

Die gewonnenen Erkenntnisse lassen sich in Anlehnung an Sesink (2015) als (potenzielle) «Wirkungen» einer mediendidaktischen Praxis zur Entwicklung medienpädagogisch relevanter Fähigkeiten in der Lehrerinnenund Lehrerbildung verstehen. Die entwickelten Lehr- und Lernsituationen mit Wikibooks eröffneten vielfältige Möglichkeiten, um das Lernen mit und über soziale Medien zur medienpädagogischer Professionalisierung erfahrungs- und reflexionsbasiert zu fördern und zu unterstützen. Dafür wurden Prozesse, ihre Rahmenbedingungen und die sich ergebenden Spannungsverhältnisse zur Gestaltung und Wahrnehmung von entsprechenden Lerngelegenheiten empirisch fundiert beschrieben.

Mit der Durchführung einer entwicklungsorientierten Bildungsforschung sind die Ergebnisse als Fallstudie hinsichtlich ihres Geltungsbereichs auf verschiedene Ebenen limitiert. Der von Sesink (2015) verwendete Wirkungsbegriff wird beispielsweise von Tulodziecki u.a. (2013) hinsichtlich seiner Potenzialität kritisiert. So stellen die Ergebnisse keine Wirkungen im Sinne von Petko (2011) oder Tulodziecki u.a. (2013) dar, weil u.a. auf die Verwendung quantitativer Tests verzichtet wurde. Zugleich können die vorgelegten Prozessbeschreibungen als Orientierungshilfe dienen, um entsprechende Tests zu entwickeln. Mit der Wahl von Wikibooks als exemplarische öffentliche Sharing-Community ist eine weitere Limitation verbunden. Interaktionen mit der Wikibook-Community erfolgten überwiegend aufgrund formaler Probleme hinsichtlich der sachgerechten Nutzung des Wiki-Editors. Es stellt sich u.a. die Frage, inwiefern andere Themen bzw. Communitys verstärkt fachliche (statt medienbezogene) Diskussionsanlässe anregen. Weiterführende Forschungsperspektiven eröffnen sich in diesem Zusammenhang für die Analyse medienbezogener Entwicklungsprozesse bei der kollaborativen Erstellung offener Lehrbücher in anderen Fachkontexten sowie in anderen öffentlichen Sharing-Communitys. 
Die skizzierten empirischen Ergebnisse bieten zudem Anknüpfungspunkte an die Diskussion zum Lernen mit sozialen Medien in der Hochschule (Mayrberger 2019, Rau 2017a). So zeigte sich in den Ergebnissen, dass das Stufenmodell zum partizipativen Lernen von Mayrberger (2019, 97) geeignet ist, um spezifische Lernsituationen analytisch einschätzen und verorten zu können. Zum anderen bieten die empirischen Ergebnisse zu den Öffnungs- und Entgrenzungsphänomen die Möglichkeit, den Modellentwurf zur partizipativen Didaktik von Mayrberger (2019) zu diskutieren. Wie am Beispiel von Wikibooks gezeigt wurde, ermöglicht die Teilhabe an öffentlichen Sharing-Communities Kommunikations- und Interaktionsmöglichkeiten mit Menschen ausserhalb des institutionellen Bildungskontextes und kann als exemplarische Ausgestaltung eines «Partizipationsraumes» (Mayrberger 2019, 192 ff.) verstanden werden, welcher ein Lernen mit und über Medien ermöglicht. Das Interessante an eben jenen Interaktionen mit der Wikibook-Community ist, dass diese einerseits zum Ausgangspunkt studentischer Entwicklungs- und Reflexionsprozesse werden können. Anderseits wurde in den zwei Fallstudien sichtbar, dass die Interaktionen mit Wikibookianerinnen und Wikibookianern in einem unterschiedlichen Ausmass erfolgten und nicht von allen Studierenden in gleicher Weise wahrgenommen wurden. Die Modellierung dieses «erweiterten» Partizipationsraumes kann eine interessante Perspektive für ein mediendidaktisches Planungsraster (Mayrberger 2019, 202 ff.) darstellen, um potenzielle Interaktionspartnerinnen und -partner jenseits des institutionellen Vermittlungszusammenhangs bei der Gestaltung digitaler Lernumgebungen berücksichtigen zu können. Ein so adaptiertes Planungs- und Verlaufsmodell kann eine vielsprechende Orientierungshilfe zur Entwicklung von Lehr- und Lernsituationen mit und über Medien im Kontext einer partizipativen Medienkultur bieten. 


\section{Literatur}

van Ackeren, Isabell, Stefan Aufenanger, Birgit Eickelmann, Steffen Friedrich, Rudolf Kammerl, Julia Knopf, Kerstin Mayrberger, Heike Scheika, Katharina Scheiter, und Mandy Schiefner-Rohs. 2019. "Digitalisierung in der Lehrerbildung. Herausforderungen, Entwicklungsfelder und Förderung von Gesamtkonzepten.» Die Deutsche Schule 111 (2019): 103-119. https://doi.org/10.31244/ dds.2019.01.10.

AG Erziehungswissenschaft. 1997. «Dokumentation der Arbeitsgruppe „Erziehungswissenschaft" von Bardo Herzig» In Neue Medien - neue Aufgaben für die Lehrerausbildung: Tagungsdokumentation, hrsg. v. Gerhard Tulodziecki und Sigrid Blömke. 39-53. Gürtersloh: Bertelsmann Stifitung.

Aßmann, Sandra, und Bardo Herzig. 2015. «Integrative Medienbildung in der Geschichtsdidaktik am Beispiel von TwHistory-Projekten.» In Medien machen Geschichte. Neue Anforderungen an den geschichtsdidaktischen Medienbegriff im digitalen Wandel, hrsg. v. Christoph Pallaske. 67-84. Berlin: Logos Verlag.

Biermann, Ralf, Johannes Fromme, und Dan Verständig. Hrsg. 2014. Partizipative Medienkulturen: Positionen und Untersuchungen zu veränderten Formen öffentlicher Teilhabe. Wiesbaden: Springer VS.

BMBF, Bundesministerium für Bildung und Forschung. 2010. «Kompetenzen in einer digital geprägten Kultur. Medienbildung für die Persönlichkeitsentwicklung, für die gesellschaftliche Teilhabe und für die Entwicklung von Ausbildungsund Erwerbsfähigkeit.» https://www.dlr.de/pt/Portaldata/45/Resources/a_dokumente/bildungsforschung/Medienbildung_Broschuere_2010.pdf

Bonk, Curtis J., Lee, Mimi M., Kim, Nari, und Meng-Fen G. Lin. 2009. «The tensions of transformation in three cross-institutional wikibook projects.» The Internet and Higher Education 12 (3-4): 126-135. https://doi.org/10.1016/j.iheduc.2009.04.002.

Flick, Uwe 2011. Triangulation: eine Einführung. 3. aktualisierte Auflage. Wiesbaden: VS Verlag für Sozialwissenschaften.

Grell, Petra, und Franco Rau. 2011. «Partizipationslücken - Social Software in der Hochschullehre.» MedienPädagogik: Zeitschrift für Theorie und Praxis der Medienbildung 21 (2011): 1-23, https://doi.org/10.21240/mpaed/21/2011.11.21.X.

Herzig, Bardo, und Grafe, Silke. 2007. Digitale Medien in der Schule: Standortbestimmung und Handlungsempfehlungen für die Zukunft. Bonn: Deutsche Telekom.

Iske, Stefan, und Winfried Marotzki. 2010. «Wikis: Reflexivität, Prozessualität und Partizipation.» In Medienbildung in neuen Kulturräumen. Die deutschprachige und britische Diskussion, hrsg. v. Ben Bachmair. 141-151. Wiesbaden: Springer VS.

Kammerl, Rudolf. 2015. «Medienbildung - (k)ein Unterrichtsfach? Eine Expertise zum Stellenwert der Medienkompetenzförderung in Schulen». https://www.mahsh.de/infothek/publikationen/medienkompetenz-expertisen.html?file=files/ infothek/publikationen/Medienpaedagogik\%2Oin\%20der\%20Kita\%202018/Medienpaedagogik_Kita_web.pdf. 
Kammerl, Rudolf, und Jönna Atzeroth, 2013. «Informationskompetenz mit modernen Schulbibliotheken fördern - Beobachtungen von Beispielen für Informationskompetenzförderung und Integration digitaler Medien im Modellprojekt "Schulbibliotheken für alle Schulen".» In Digitale Medien und Schule. Zur Rolle digitaler Medien in Schulpädagogik und Lehrerbildung, hrsg. v. Dietrich Karpa, Birgit Eickelmann, und Silke Grafe. 95-109. Immenhausen: Prolog.

Kammerl, Rudolf, und Kerstin Mayrberger. 2011. «Medienpädagogik in der Lehrerinnen- und Lehrerbildung in Deutschland: Aktuelle Situation und Desiderata.» Beiträge zur Lehrerinnen- und Lehrerbildung 29 (2011): 172-184.

Kammerl, Rudolf, und Sandra Ostermann. 2010. «Medienbildung - (k)ein Unterrichtsfach? Eine Expertise zum Stellenwert der Medienkompetenzförderung in Schulen». https://www.ma-hsh.de/infothek/publikationen/medienkompetenz-expertisen.html?file=files/infothek/publikationen/Medienpaedagogik\%2Oin\%2Oder\%2OKita\%2O2018/web\%2OMA\%2OHSH_Studie\%2OMedienbildung_180210.pdf.

KMK. Kultusministerkonferenz 2017. «Bildung in der digitalen Welt. Strategie der Kultusministerkonferenz». Beschluss der Kultusministerkonferenz vom 08.12.2016 in der Fassung vom 07.12.2017 https://www.kmk.org/fileadmin/Dateien/veroeffentlichungen_beschluesse/2018/Strategie_Bildung_in_der_digitalen_Welt_idF._vom_07.12.2017.pdf.

KMK. Kultusministerkonferenz 2019a. «Ländergemeinsame inhaltliche Anforderungen für die Fachwissenschaften und Fachdidaktiken in der Lehrerbildung». Beschluss der Kultusministerkonferenz vom 16.12.2004 i. d. F. vom 16.05.2019 https://www.kmk.org/fileadmin/Dateien/veroeffentlichungen beschluesse/2008/2008_10_16-Fachprofile-Lehrerbildung.pdf.

KMK. Kultusministerkonferenz 2019b. «Standards für die Lehrerbildung: Bildungswissenschaften». Beschluss der Kultusministerkonferenz vom 16.12.2004 i. d. F. vom 16.05.2019 https://www.kmk.org/fileadmin/veroeffentlichungen_beschluesse/2004/2004_12_16-Standards-Lehrerbildung-Bildungswissenschaften.pdf.

Kuckartz, Udo, Dresing, Thorsten, Rädiker, Stefan, und Stefer, Claus 2008. Qualitative Evaluation: der Einstieg in die Praxis. 2. aktualisierte Auflage. Wiesbaden: Springer VS.

Kühnel, Steffen, und Dagmar Krebs 2006. Statistik für die Sozialwissenschaften: Grundlagen, Methoden, Anwendungen. 3. Auflage. Reinbek bei Hamburg: Rowohlt.

Mayrberger, Kerstin. 2012. «Medienpädagogische Kompetenz im Wandel - Vorschlag zur Gestaltung des Übergangs in der Lehrerbildung am Beispiel mediendidaktischer Kompetenz.» In Jahrbuch Medienpädagogik 9. Methodologie und Methoden medienpädagogischer Forschung, hrsg. v. Renate Schulz-Zander, Birgit Eickelmann, Heinz Moser, Horst Niesyto und Petra Grell. 389-412. Wiesbaden: Springer VS. 
Mayrberger, Kerstin. 2013. «Partizipatives Lernen mit dem Social Web in der Schule.» In Organisation und Partizipation: Beiträge der Kommission Organisationspädagogik, hrsg. v. Susanne M. Weber, Andreas Schröder, Claudia Fahrenwald und Hildegard Macha. 167-175. Wiesbaden: Springer VS.

Mayrberger, Kerstin. 2019. Partizipative Mediendidaktik. Gestaltung der (Hochschul-) Bildung unter den Bedingungen der Digitalisierung. Weinheim u. Basel: Beltz Juventa.

Mayrberger, Kerstin, und Heinz Moser. 2011. «Editorial: Partizipationschancen im Kulturraum Internet nutzen und gestalten: Das Beispiel Web 2.0» MedienPädagogik: Zeitschrift für Theorie und Praxis der Medienbildung 21 (2011). https://doi. org/10.21240/mpaed/21/2011.10.10.X.

Mayrberger, Kerstin, Stephan Waba, und Michael Schratz. 2013. «Social Media in der Lehrerbildung. Editorial» Journal für LehrerInnenbildung 13 (2013), Nr. 4: 4-5.

Mayring, Philipp. 2002. Einführung in die qualitative Sozialforschung: Eine Anleitung zu qualitativem Denken. Weinheim u.a.: Beltz.

Moser, Heinz. 2010. Schule 2.0: Medienkompetenz für den Unterricht. Kronach: Link.

Niesyto, Horst. 2014. «Medienpädagogische Praxisforschung.» In Jahrbuch Medienpädagogik. Methodologie und Methoden medienpädagogischer Forschung, hrsg. v. Anja Hartung, Bernd Schorb, Horst Niesyto, Heinz Moser und Petra Grell. Bd. 10. 173-101. Wiesbaden: Springer VS.

Petko, Dominik. 2011. «Praxisorientierte medienpädagogische Forschung: Ansätze für einen empirischen Perspektivenwechsel und eine stärkere Konvergenz von Medienpädagogik und Mediendidaktik.» MedienPädagogik: Zeitschrift für Theorie und Praxis der Medienbildung 20 (2011): 245-258. https://doi.org/10.21240/ mpaed/20/2011.09.22.X.

Rau, Franco. 2017a. «Hochschullehre mit dem Social Web? Zur Einschätzung von Trendthemen in Bildungskontexten.» In «Trendy, hip und cool». Auf dem Weg zu einer innovativen Hochschule? hrsg. v. Diana Bücker u.a. 95-105. Bielefeld: Bertelsmann Verlag.

Rau, Franco. 2017b. «Interaktives und kollaboratives Lernen mit sozialen Medien? Spannungsfelder in der Hochschullehre.» In Lehren und Lernen online: Lehr- und Lernerfahrungen im Kontext akademischer Online-Lehre, hrsg. v. Hedwig R. Griesehop, und Edith Bauer, 131-148. Wiesbaden: Springer VS.

Rau, Franco. 2020. Lernsituationen mit Metaphern und Wikibooks. Fallstudien zu Entwicklungspotenzialen einer integrativen Medienbildung in der Lehrerinnen- und Lehrerbildung. Zürich: MedienPädagogik: Zeitschrift für Theorie und Praxis der Medienbildung. https://doi.org/10.21240/mpaed/diss.fr.X.

Reinmann, Gabi, und Werner Sesink. 2014. «Begründungslinien für eine entwicklungsorientierte Bildungsforschung». In Jahrbuch Medienpädagogik 10. Methodologie und Methoden medienpädagogischer Forschung, hrsg. v. Anja Hartung, Bernd Schorb, Horst Niesyto, Heinz Moser und Petra Grell. 75-89. Wiesbaden: Springer VS. 
Schiefner-Rohs, Mandy. 2012. Kritische Informations- und Medienkompetenz Theoretisch-konzeptionelle Herleitung und empirische Betrachtungen am Beispiel der Lehrerausbildung. Münster u.a.: Waxmann.

Sesink, Werner. 2015. Entwicklungsorientierte Bildungsforschung. Plädoyer für einen "dritten Weg" in pädagogischer Forschung. Eine Textsammlung. http://www.sesink.de/wordpress/wp-content/uploads/2015/11/Entwicklungsorientierte-Bildungsforschung_Sesink_2015.pdf.

Sesink, Werner, und Gabi Reinmann. 2015. «Umrisse eines Strukturmodells für entwicklungsorientierte bildungswissenschaftliche Forschung». In Entwicklungsorientierte Bildungsforschung. Plädoyer für einen "dritten Weg" in pädagogischer Forschung. Eine Textsammlung, hrsg. v. Werner Sesink. 69-83 http://www. sesink.de/wordpress/wp-content/uploads/2015/11/EntwicklungsorientierteBildungsforschung_Sesink_2015.pdf.

Sim, Jeffrey Wee S., und Khe F. Hew .2010. «The use of weblogs in higher education settings: A review of empirical research» Educational Research Review 5 (2010), Nr. 2: 151-163. https://doi.org/10.1016/j.edurev.2010.01.001.

Spanhel, Dieter. 2007. «Zur Standortbestimmung der Medienpädagogik aus anthropologischer und bildungswissenschaftlicher Sicht.» In Jahrbuch Medienpädagogik. Standortbestimmung einer erziehungswissenschaftlichen Disziplin, hrsg. v. Werner Sesink, Michael Kerres, und Heinz Moser. Bd. 6. 33-54. Wiesbaden: Springer VS.

Spannagel, Chrstian. 2013. «Am Modell lernen: Produktive Nutzung digitaler Medien im Lehramtsstudium » Journal für LehrerInnenbildung 13 (2013), Nr. 4: 12-16.

Spannagel, Chrstian, und Florian Schimpf. 2009. «Öffentliche Seminare im Web 2.0». In Lernen im Digitalen Zeitalter. Workshop-Band Dokumentation der PreConference zur DeLFI2009, hrsg. v. Andreas Schwill und Nicolas Apostolopulos. 13-20. Potsdam und Berlin: Logos Verlag.

Tulodziecki, Gerhard, Bardo Herzig, und Silke Grafe. 2013. Gestaltungsorientierte Bildungsforschung und Didaktik: Theorie - Empirie - Praxis. Bad Heilbrunn: Klinkhardt.

Wildt, Johannes. 2002. «Ein hochschuldidaktischer Blick auf Lehren und Lernen.» In Neues Handbuch Hochschullehre, hrsg. v. Brigitte Berendt, Hans-Peter, Voss und Johannes Wildt. 1-10. Bonn: Raabe-Verlag.

Wildt, Johannes. 2013. "Entwicklung und Potentiale der Hochschuldidaktik.»In Professionalisierung der Lehre. Perspektiven formeller und informeller Entwicklung von Lehrkompetenz im Kontext der Hochschulbildung, hrsg. v. Johannes Wildt und Matthias Heiner. 27-57. Bielefeld: Bertelsmann.

Xiao, Yun, und Robert Lucking. 2008. «The impact of two types of peer assessment on students' performance and satisfaction within a Wiki environment» The Internet and Higher Education 11 (3-4): 186-193. https://doi.org/10.1016/j.iheduc.2008.06.005. 